\title{
La vacunación frente al sarampión en profesionales de la salud
}

\author{
P. Díaz Pérez \\ A Global Perspective of Vaccination of Healthcare Personnel against Measles: Systematic Review \\ Vaccine, vol. 32, no. 38. USA / Agosto 2014 \\ Amy Parker Fiebelkorn, Jane F. Seward and Walter Orenstein \\ DOI: 10.1016/j.vaccine.2013.11.005
}

DOI: 10.12961/aprl.2019.22.04.05

\section{RESUMEN}

La transmisión del sarampión en establecimientos sanitarios ha sido bien documentada. El personal sanitario no vacunado y que carece de otras pruebas de inmunidad contra el sarampión se pone en riesgo a sí mismo y a sus pacientes de contraer el sarampión. Se realizó una revisión bibliográfica sistemática de las políticas de vacunación contra el sarampión y su implementación en el personal sanitario, la seroprevalencia del sarampión entre el personal sanitario, la transmisión del sarampión y la carga de enfermedades en los entornos sanitarios, y el impacto/costes generados por los centros sanitarios debido a la transmisión del sarampión asociado a la atención sanitaria. Las búsquedas en cinco bases de datos identificaron 135 artículos relevantes; se encontraron 47 artículos adicionales a través de referencias cruzadas. Se estima que el riesgo de contraer sarampión es de 2 a 19 veces mayor para el personal sanitario susceptible que para la población general. Durante el período 1989-2013 cincuenta y tres artículos publicados en todo el mundo informaron sobre la transmisión del sarampión de pacientes al personal de salud; muchos de los profesionales de la salud no estaban vacunados o su estado de vacunación era desconocido. Dieciocho artículos publicados en todo el mundo durante 1982-2013 describieron ejemplos de transmisión de profesionales sanitarios a pacientes o a otro personal sanitario. La mitad de los países europeos no tienen políticas de vacunación contra el sarampión para el personal sanitario. No hay recomendaciones de política global sobre la vacunación del personal sanitario contra el sarampión. Incluso en países como Estados Unidos o Finlandia que tienen políticas nacionales, las recomendaciones no se aplican de manera uniforme en los centros sanitarios. La serosusceptibilidad al sarampión en el personal de la salud varió ampliamente entre los estudios (mediana 6,5\%, rango 0\%-46\%) pero fue consistentemente mayor entre el personal sanitario más joven. Las deficiencias en la documentación de la administración de dos dosis de vacunación contra el sarampión u otra evidencia de la inmunidad entre el personal sanitario presentan desafíos para responder a la exposición al sarampión en entornos de la salud. La evaluación y la contención de exposiciones y brotes en entornos sanitarios pueden ser disruptivas y costosas. Establecer políticas de vacunación contra el sarampión para el personal sanitario es una estrategia

Sección coordinada por:

Consol Serra (consol.serra@upf.edu)

$\mathrm{M}^{\mathrm{a}}$ del Mar Seguí (mm.segui@ua.es)

Arch Prev Riesgos Labor 2019; 22 (4): 178-180 importante para lograr la eliminación del sarampión y debe ser una alta prioridad para el establecimiento de políticas globales, grupos, gobiernos y hospitales.

\section{COMENTARIO}

El sarampión es una enfermedad vírica aguda muy contagiosa, que se transmite por vía aérea y se caracteriza por la aparición de exantema y sintomatología respiratoria febril. En España es una enfermedad de declaración urgente, prevenible y con la tasa más alta de reproducción que cualquiera del resto de enfermedades prevenibles mediante la vacunación.

La primera vacuna frente al sarampión se autorizó en España en el año 1965 y se suspendió en 1970 debido a la elevada incidencia de reacciones adversas. No fue hasta el año 1978 cuando se incluyó la vacuna del sarampión monocomponente a los 9 meses de edad, sustituida en 1981 por la vacuna triple vírica frente a sarampión, rubéola y parotiditis con una dosis a los 15 meses. En 1996 se instauró la vacuna triple vírica con una pauta de dos dosis. Y en 2012 el Consejo Interterritorial del Sistema Nacional de Salud estableció que la primera dosis de vacuna triple vírica (TV) se administrase a los 12 meses de edad y la segunda dosis entre los 3-4 años de edad'.

En 2017, la OMS-Europa declaró que España había eliminado el sarampión tras un periodo de 36 meses sin transmisión endémica $(2014,2015$ y 2016), pero hubo y sigue habiendo casos confirmados posteriores debidos o relacionados con la importación de la infección y la disminución de la cobertura de vacunación (coberturas con dos dosis de TV por debajo del objetivo del $95 \%$ e incluso dos Comunidades Autónomas por debajo del 90\%).

El sarampión se ha diagnosticado sobre todo en adultos entre los 20-49 años no vacunados. En estos grupos de edad, el 21,9\% de los casos había recibido dos dosis de vacuna con una media de 20,3 años transcurridos desde la última dosis ${ }^{1}$.

Se considera que el riesgo de trasmisión del sarampión para el personal sanitario es hasta 13 veces mayor que el de la población general $^{2}$. Además, el personal sanitario ha tenido un papel relevante en la transmisión de brotes ocurridos en los últimos años en España ${ }^{3}$. En este sentido, de los 26 brotes ocurridos en Cataluña en el período 2001-2013, con 797 casos en total, el 72,5\% de ellos fueron en trabajadores sanitarios (médicos y personal de enfermería) y el $22,5 \%$ otros profesionales relacionados con el ámbito sanitario ${ }^{4,5}$. También en Madrid, en un brote registrado en 2006, un $60 \%$ de los casos eran adultos y un $15 \%$ de estos eran perso- 
nal sanitario ${ }^{6}$. En 2017, en 5 brotes se identificó la transmisión en hospitales, con afectación tanto en sanitarios como pacientes ${ }^{1}$. Respecto al gran número de brotes detectados, se ha descrito que la trasmisión nosocomial ocurre principalmente en las salas de espera de centros sanitarios, en las zonas de urgencias pediátricas y urgencias generales, y en áreas de hospitalización de pediatría. Entre los profesionales sanitarios, los médicos internos residentes son los más frecuentemente implicados en los brotes de sarampión.

La prevención de enfermedades infecciosas es un tema prioritario en la política de salud pública de cualquier país y, en concreto, la vacunación, por ser una de las medidas de prevención que ha demostrado ser más coste-efectiva para la mejora de la salud y el bienestar general de la población. A pesar de que cada país diseñe una política de vacunación en base a su situación epidemiológica distintiva y sus objetivos políticos particulares, tanto la Organización Mundial de la Salud (OMS) como el Fondo de las Naciones Unidas para la Infancia (UNICEF) han desarrollado estrategias o planes sobre la inmunización y prevención de la población a nivel global. En 2012 la Asamblea de la OMS aprobó el Plan de Acción Mundial sobre Vacunas 2011-20207, donde se proponen distintos retos, entre ellos eliminar el sarampión y la rubéola.

En la Unión Europea (UE) no existe un órgano legislativo supranacional con competencia para regular la política de vacunación de todos los Estados Miembros de forma homogénea. Sin embargo, sí participa activamente en la política de Salud Pública a través de la definición de los "Programas europeos de salud". Por tanto, al no existir un modelo de prevención común en la UE se originan desigualdades en el acceso a la inmunización entre ciudadanos comunitarios. De hecho, la OMS ha alertado recientemente sobre la seria amenaza que supone la difusión del virus del sarampión en territorio europeo, indicando que ningún Estado se encuentra al margen del mismo ${ }^{12}$. Entre octubre de 2017 y septiembre de 2018, 30 Estados Miembros notificaron 13.453 casos de sarampión, 9.389 (70\%) de los cuales fueron confirmados por laboratorio. Si tenemos en cuenta los países que más casos confirmados obtuvieron, el rankig se inicia en Grecia con 3.049 casos, seguido de Francia con 2.771, Italia con 2.599, Rumania con 1.821 y el Reino Unido con 1.019 casos notificados, los que representan el 23\%, 21\%, 19\%, 14\% y $8 \%$ de todos los casos, respectivamente. De estos, ocurrieron 36 muertes atribuidas a sarampión ( 24 en Rumania, 6 en Italia, 4 en Grecia y 3 en Francia) ${ }^{9}$.

Merece la pena reflexionar sobre lo recientes que son las políticas de vacunación poblacionales, lo que significa que en el momento actual parte de la población adulta, justamente la anterior a dichas políticas que no se beneficiaron de esta medida de prevención, puede que no estén correctamente inmunizadas. Esta parte de la población representa unos porcentajes más o menos importantes de personas susceptibles que en algunos casos son los responsables de la persistencia de brotes de estas enfermedades que son objeto de programas de vacunación. Por tanto, se tendrían que modificar las estrategias con políticas activas y transmitirlas con mensajes eficaces que reduzcan las dudas, poniendo en valor no sólo el efecto individual de la vacuna, sino el poder de crear inmunidad colectiva ${ }^{8}$.
En la revisión bibliográfica que nos ocupa, se concluye que, a pesar del bajo riesgo de exposición al sarampión y la elevada efectividad de la vacunación para prevenir la transmisión de la infección, actualmente no existe una política global de la OMS para la vacunación del personal sanitario contra el sarampión. Es esencial definir y documentar los criterios para la evidencia de inmunidad para la implementación y evaluación de políticas de vacunación del personal sanitario. La mayoría de los artículos revisados, que informaban sobre la cobertura de vacunación, no describían si los profesionales de la salud tenían otra evidencia de inmunidad frente al sarampión, lo que hace imposible evaluar la verdadera cobertura de vacunación y el número potencial de profesionales de la salud susceptibles. Estos problemas también complicaron los estudios de interpretación sobre la susceptibilidad al sarampión en el personal de salud. Además, la determinación de $\mathrm{IgG}$ frente al sarampión varía en sensibilidad y especificidad, lo que complica las comparaciones entre los estudios. Aunque se reconoce que los criterios de evidencia de inmunidad no proporcionan una garantía del $100 \%$ para considerar a la persona "inmune" (por ejemplo, 2 dosis de la vacuna contra el sarampión no son $100 \%$ efectivas, las pruebas de laboratorio para determinación de $\lg G$ pueden tener resultados positivos y negativos falsos), son criterios útiles para la implementación de programas de inmunización en la práctica.

En la encuesta de seroprevalencia realizada en España ${ }^{13}$ se mostró que durante los años 2009, 2011 y 2013 (País Vasco, Madrid y Galicia), la seroprotección frente a sarampión en adultos era superior al 95\% en las personas nacidas antes de 1980, y del $99 \%$ en las nacidas antes de 1971, excepto en el País Vasco $(97,7 \% \text { nacidas antes de } 1971)^{13}, 14,15,16$. En un estudio realizado en Cataluña entre junio de 2008 y diciembre de 2009, se observó que la seroprotección frente a sarampión en personal sanitario era del 98\% (IC 95\% 96,6-98,9), siendo más baja en los nacidos a partir de 1981 con respecto a los nacidos entre 1965 y 1980 $(94,4 \% \text { frente a } 99 \% \text {, respectivamente })^{17}$.

Por otro lado, en caso de platear la necesidad de crear políticas obligatorias de vacunación, se debe tener en cuenta que dichas políticas pueden suponer una violación de los derechos individuales. Por lo tanto, el beneficio individual y de salud pública de la intervención debe ser claro, debe requerir una exclusión voluntaria activa o permitir exenciones por diversos motivos (creencias médicas, religiosas o de otro tipo) y debe prever alguna sanción por negarse a cumplirla. Además, los centros sanitarios deben considerar las consecuencias médicas y legales en caso de que uno de sus profesionales se niegue a vacunarse, contraiga la enfermedad y la transmita a los pacientes, especialmente a aquellos con enfermedades inmunodeprimidas que no pueden vacunarse y que sólo están protegidos contra el sarampión a través de la inmunidad de la comunidad, o la falta de exposición.

Sirva como ejemplo lo acontecido en Italia en los últimos años. Las bajas coberturas de vacunación que actualmente presenta, van acompañadas de un nivel de desconfianza en la seguridad de las vacunas por encima de la media mundial. En base al estudio “The Vaccine Confidence 2016" realizado por la London School of Hygiene and Tropical Medicine, para el cual se entrevistó a una muestra de 65.819 personas en 67 países, un 20,6\% de los italianos encuestados (por encima de la media del 12\% en los datos 
mundiales) indicaron que estaban en desacuerdo o totalmente en desacuerdo con la afirmación: "Las vacunas son seguras"10.

Italia ha optado por reforzar su política de obligatoriedad de vacunas, mediante la elaboración de una ley que aumenta el número de vacunas obligatorias para la infancia y adolescencia de 4 a 12 años. El cumplimiento de la vacunación obligatoria será necesario para que los niños puedan ser admitidos en la guardería y en los centros de educación preescolar (niños de 0 a 6 años). Por su parte, los niños de primaria podrán ser admitidos en los colegios, pero sus padres podrán incurrir en sanciones administrativas en caso de no cumplir con la vacunación obligatoria. El objetivo de esta medida no es otro que el de contrarrestar la disminución gradual que vienen sufriendo las coberturas de vacunación en los últimos años, siendo muchas de ellas actualmente inferiores al 95\%, cifra recomendada por la OMS para garantizar la inmunidad colectiva ${ }^{11}$.

Para concluir, la transmisión del sarampión en entornos sanitarios sigue siendo un grave problema de salud pública que puede tener consecuencias graves y fatales en pacientes de alto riesgo. Asimismo, los profesionales sanitarios que no están correctamente inmunizados se exponen y exponen a los usuarios del sistema sanitario al sarampión. Por tanto, implementar políticas de vacunación en profesionales sanitarios podría ser una opción efectiva y eficaz para erradicar esta enfermedad.

Por otro lado, los servicios de Salud Laboral deben conocer y registrar las coberturas de vacunación de los profesionales que trabajan en centros sanitarios, así como del grado de inmunidad de éstos profesionales, principalmente los que trabajan en áreas con un mayor riesgo de infección por el virus de sarampión, como serían los servicios de urgencias, pediatría y todos aquellos profesionales que inician la actividad laboral en un centro sanitario, con el fin de identificar a los susceptibles de contraer la enfermedad y ofrecer la vacunación.

\section{BIBLIOGRAFÍA}

1. Instituto de Salud Carlos III. CIBERESP y Ministerio de Sanidad Consumo y Bienestar Social. Plan Nacional de Eliminación del Sarampión y de la Rubeola. Informe anual 2017. Madrid, 21 de diciembre de 2018.

2. Campins M, Uriona S. Epidemiología general de las infecciones adquiridas por el personal sanitario. Inmunización del personal sanitario. Enferm Infecc Microbiol Clin. 2014;32(4):259-265. DOI: 10.1016/j.eimc.2014.02.002

3. Peña-Rey I, Martínez de Aragón V, Mosquera M, De Ory F, Echevarría JE, Measles Elimination Plan Working Group in Spain. Measles risk groups in Spain: Implications for the European measleselimination target. Vaccine. 2009; 27:3927-34

4. Torner N, Solano R, Rius C, Domínguez A and the Measles Elimination Program Surveillance Network of Catalonia, Spain. Implication of health care personnel in measles transmission. The need for updated immunization status in the move towards eradication of measles in Catalonia. Hum Vaccin Immunother. 2015; 11: 288-292.

5. Fernández-Canoa MI, Armadans L, Sulleiro E, 1 Espugad M, Ferrer E, Martínez-Gómez X et al. Susceptibilidad frente a sarampión y varicela en el personal sanitario de un hospital de tercer nivel en Cataluña. Enferm Infecc Microbiol Clin. 2012;30(4):184-188.

6. Garcia-Comas L. Measles outbreak in the region of Madrid, Spain, 2006. Euro Surveill. 2006;11(13):pii=2935. Disponible en: http://www.eurosurveillance.org/ViewArticle. aspx?Articleld=2935.

7. Plan de Acción Mundial sobre Vacunas 2011-2020. 2013.

8. Las vacunas en España. Situación actual y perspectiva de futuro. Noviembre 2017.

9. ECDC. Monthly measles and rubella monitoring. Web Corporativa ECDC. 09 Nov 2018. https://ecdc.europa.eu/sites/portal/files/documents/measlesrubella-monthly-monitoring-report-november-2018.pdf.

10. The Vaccine Confidence Project. The State of Vaccine Confidence. Web Corporativa The Vaccine Confidence Project. [En línea] 2016. http://www.vaccineconfidence.org/research/the-state-of-vaccineconfidence-2016/.

11. Ministero della Salute. Home- Decreto legge vaccini. Web Corporativa Ministero della Salute. [En línea] 17 de Agosto de 2017. http://www.salute.gov.it/ portale/vaccinazioni/dettaglioContenutiVaccinazioni. jsp?lingua=italiano\&id= $4824 \&$ area $=$ vaccinazioni $\&$ menu $=$ vuoto.

12. Naciones Unidas. Noticias- Brote de Sarampión en Europa amenaza eliminación de la enfermedad. Web Corporativa Naciones Unidas. [En línea] 28 de Marzo de 2017. http://www.un.org/ sustainabledevelopment/es/2017/03/brote-de-sarampion-en-europa-amenaza-eliminacion-de-laenfermedad/.

13. Grupo de trabajo de la Ponencia de Programa y Registro de Vacunaciones. Vacunación en trabajadores sanitarios. Comisión de Salud Pública del Consejo Interterritorial del Sistema Nacional de Salud. Ministerio de Sanidad, Servicios Sociales e Igualdad, 2017. https://www.mscbs.gob.es/profesionales/saludPubli$\mathrm{ca} /$ prevPromocion/vacunaciones/docs/Vacunacion_sanitarios.pdf.

14. I Encuesta de seroprevalencia de la Comunidad Autónoma del País Vasco. Departamento de Sanidad y Consumo. Gobierno Vasco. 2011.

15. García Comas L, Ordobás M, Sanz JC, Ramos B, García J, Cevallos C, Verdejo J, Barranco D, Astray J, Echevarría JM, Ortiz M, del Amo J, Moreno S. IV Encuesta de serovigilancia de la Comunidad de Madrid. Consejería de Sanidad. Dirección General de Atención Primaria. Documento Técnico de Salud Pública. Madrid 2015.

16. Encuesta gallega de seroprevalencia 2013. Boletín epidemiológico de Galicia. Vol. XXVI-nº 4. Septiembre 2014.

17. Urbiztondo L, Borrás E, Costa J, Broner S, Campins M, Bayas JM et al. Prevalence of measles antibodies among health care worker in Catalonia (Spain) in the elimination era. BMC Infectious Diseases 2013, 13:391.

18. De Ory F, Peña-Rey I. Sarampión en la línea de meta de la eliminación. Enferm Infecc Microbiol Clin. 2010;28:73-74.
Pilar Díaz Pérez

Servicio de Salud Laboral, Parc de Salut Mar, Barcelona pdiazperez@parcdesalutmar.cat 